\title{
Advantages of Strong Mode Coupling for Suppression of Nonlinear Distortion in Few-Mode Fibers
}

\author{
Filipe M. Ferreira, Naoise Mac Suibhne, Christian Sánchez, Stylianos Sygletos, Andrew Ellis \\ Aston Institute of Photonic Technologies, Aston University, Birmingham, B4 7ET, UK \\ f.ferreira@aston.ac.uk
}

\begin{abstract}
Nonlinear distortion in few-mode fibers for intermediate coupling is studied for the first time. Coupling strengths beyond $-20 \mathrm{~dB} / 100 \mathrm{~m}$ give suppression of nonlinear distortion below the isolated mode without mode coupling.

OCIS codes: (060.0060) Fiber optics and optical communications; (060.4370) Nonlinear optics, fibers.
\end{abstract}

\section{Introduction}

In principle, an $N$-fold capacity increase can be obtained by using one FMF guiding $N$ independent modes in place of one single-mode fiber (SMF). However, FMFs present new impairments that have to be addressed in order to reach their full capacity [1]-[4] including; linear mode coupling, differential mode delay, and inter-mode nonlinear effects. Linear coupling plays a key role since stronger coupling acts to lower the overall group delay spread [2] and the intermode nonlinear distortion [5]. However whilst nonlinear inter-mode interactions have been experimentally measured [3] and numerical models have been made available [4]-[7], to the best of our knowledge the nature of the dependence of the nonlinear distortion on the coupling strength is not fully understood in the intermediate regime.

In this paper, we evaluate the dependence of the nonlinear distortion on the linear mode coupling strength through exhaustive stepwise simulation [7],[8], considering a particular FMF supporting four non-degenerate linearly polarized (LP) modes for simplicity. In particular our simulations show that with no mode coupling, nonlinear distortion is increased by inter-mode contributions, but by increasing the coupling strengths the nonlinear distortions reduce, gradually erasing the observed step like profile [9] and beyond $-20 \mathrm{~dB} / 100 \mathrm{~m}$ a suppression of nonlinear distortion below the isolated mode without mode coupling can be achieved.

\section{Analysis}

The commercial use of FMF in long haul systems is likely to require the use of a substantial fraction of the bandwidth. In such case, the number of inter-mode interactions that are velocity-matched increases significantly, especially in fibers with low differential mode delay (required for low complexity digital signal processing). For FMFs, velocity matching may be achieved by balancing the two dispersive processes present, the differential mode delay and the chromatic dispersion [3].

The generalization of the single-mode four-wave mixing (FWM) theory to a multi-mode fiber has been shown to be straightforward [4]-[6]. When considering three waves denoted $p, q, r$ propagating in modes denoted $a, b, c$, respectively, the nonlinear signal field $E_{d s}$ generated at angular frequency $\omega_{s}=\omega_{p}+\omega_{q}-\omega_{r}$ in mode $d$ is:

$$
E_{d s}=\gamma_{a b c d} E_{a p} E_{b q} E_{c r}^{*}\left(1-e^{-\alpha L} e^{-\mathrm{j} \Delta \beta_{a b c d, p q r s} L}\right) /\left(\mathrm{j} \Delta \beta_{a b c d, p q r s} L+\alpha\right) \cdot e^{-\alpha / 2 L} e^{-\mathrm{j} \beta_{d s} L}
$$

where $\alpha$ is the attenuation, $L$ is the span length, $\gamma_{a b c d}$ is the nonlinear coefficient for the interaction between modes $a, b, c, d$, and $\Delta \beta_{a b c d, p q r s}$ is the phase mismatch between waves $p, q, r, \mathrm{~s}$. The phase mismatch is given by $\Delta \beta_{a b c d, p q r s}=\beta_{a p}+\beta_{b q}-\beta_{c r}-\beta_{d s}$ where $\beta_{a p}$ is the propagation constant of mode $a$ at angular frequency $\omega_{p}$.

For an optical super-channel with a total bandwidth $B$ the total nonlinear noise generated by FWM between a given set of modes may be calculated by integrating the product of (1) with the signal power spectral density (PSD) in each mode. A closed form solution for this integral was obtained (and experimentally validated) for the case of a signal with a rectangular spectrum (OFDM or Nyquist WDM super channel) in each interacting mode, and the overall efficiency parameter $\eta_{a b c d}$ was shown to be [9]:

with:

$$
\eta_{a b c d}=\frac{\gamma_{a b c d}^{2}}{\pi \alpha\left|\beta^{(2)}\right|}\left[\ln \left(\frac{B^{2}+2 B \Delta f_{a b c d}}{2 f_{w}}\right)+s \ln \left(s \frac{B^{2}-2 B \Delta f_{a b c d}}{2 f_{w}}\right)\right],
$$

$$
\begin{aligned}
& \Delta f_{a b c d}=\left(\beta_{a}^{(1)}+\beta_{b}^{(1)}-\beta_{c}^{(1)}-\beta_{d}^{(1)}\right) / 2 \pi \beta^{(2)} ; \\
& s=\operatorname{sign}\left(B-2 \Delta f_{a b c d}\right) ; f_{w}=\sqrt{\alpha / 4 \pi^{2}\left|\beta^{(2)}\right|}
\end{aligned}
$$




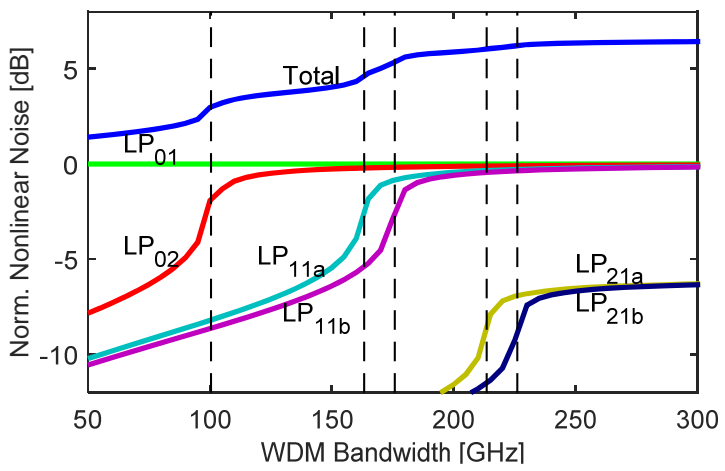

Table 1. Characteristics of the four LP mode FMF [11].

\begin{tabular}{c} 
Attenuation [dB/km]: 0.2 \\
\begin{tabular}{|l|l|l|l|l|l|l|}
\hline & Differential Mode Delay $[\mathrm{ps} / \mathrm{km}]$ \\
\hline & $\mathrm{LP}_{01}$ & $\mathrm{LP}_{02}$ & $\mathrm{LP}_{11 \mathrm{a}}$ & $\mathrm{LP}_{1 \mathrm{~b}}$ & $\mathrm{LP}_{21 \mathrm{a}}$ & $\mathrm{LP}_{21 \mathrm{~b}}$ \\
\hline $\mathrm{LP}_{01}$ & 0 & 8 & 13 & 14 & 17 & 18 \\
\hline
\end{tabular} \\
\hline \\
LP Chromatic Dispersion [ps/nm/km] \\
\hline
\end{tabular}

Effective Area $\left[\mu \mathrm{m}^{2}\right]$
\begin{tabular}{|l|l|l|l|l|l|l|}
\hline & $\mathrm{LP}_{01}$ & $\mathrm{LP}_{02}$ & $\mathrm{LP}_{11 \mathrm{a}}$ & $\mathrm{LP}_{11 \mathrm{~b}}$ & $\mathrm{LP}_{21 \mathrm{a}}$ & $\mathrm{LP}_{21 \mathrm{~b}}$ \\
\hline $\mathrm{LP}_{01}$ & 146 & 291 & 291 & 291 & 583 & 583 \\
\hline $\mathrm{LP}_{02}$ & 291 & 290 & 578 & 578 & 578 & 578 \\
\hline $\mathrm{LP}_{11 \mathrm{a}}$ & 291 & 578 & 193 & 579 & 386 & 386 \\
\hline $\mathrm{LP}_{11 \mathrm{~b}}$ & 291 & 578 & 579 & 193 & 386 & 386 \\
\hline $\mathrm{LP}_{21 \mathrm{a}}$ & 583 & 578 & 386 & 386 & 257 & 772 \\
\hline $\mathrm{LP}_{21 \mathrm{~b}}$ & 583 & 578 & 386 & 386 & 772 & 257 \\
\hline
\end{tabular}

Fig. 1. Contribution to the nonlinear noise power in the $\mathrm{LP}_{01}$ mode for signals propagating in higher order modes normalized by the $\mathrm{LP}_{01}$ intra-

modal nonlinear noise power, as a function of WDM bandwidth.

where $\beta_{a}^{(1)}$ is the group delay of mode $a, \beta^{(2)}$ is the second order dispersion coefficient, and $\Delta f_{a b c d}$ is the velocitymatched frequency offset. All modes are assumed to have identical second order dispersion coefficients $\beta^{(2)}$ and frequency independent mode group velocities $\beta_{a}^{(1)}$. Note that the FWM efficiency is maximized at a velocity-matched frequency offset $\Delta f_{a b c d}$ where chromatic dispersion and differential mode delay cancel exactly. Since it is also necessary to cancel the contributions due to $\beta_{a}^{(0)}$ in this paper strong inter-mode phase-matching is only considered for interactions of pairs of modes $(a=d, b=c$ or $b=d, a=c)$ [10]. Finally, the total nonlinear power generated in mode $d$ is given by $\left(\sum_{a, b, c} \eta_{a b c d}\right) P_{a} P_{b} P_{c}$, where $P_{a}$ is the signal power spectral density in mode $a$.

Fig. 1 shows the nonlinear noise power generated at the center of the WDM signal as a function of the overall bandwidth for a particular four LP mode fiber with no linear mode coupling. The fiber considered has a graded-index core with a refractive index relative difference of $4.5 \times 10^{-3}$ and a radius of $12.83 \mu \mathrm{m}$, optimized in [11] for low DMD. The fiber characteristics are presented in Table 1. In Fig. 1, a number of discontinuities are apparent in the total nonlinear noise, in addition to the logarithmically increasing background expected for a SMF [12]. These discontinuities correspond to the signal bandwidth $(B)$ becoming sufficiently large to result in an additional strongly velocity matched interactions. In Fig. 1 , the dashed vertical lines identify these points $\left(B / 2=\left|\Delta f_{a b c d}\right|\right)$. As the WDM bandwidth is increased, a higher number of strong inter-mode nonlinear contributions are accumulated.

\section{Results}

The analysis in the last section was performed assuming the weak coupling regime. However, the majority of the FMFs presented up now [13] show higher crosstalk, ranging from $-50 \mathrm{~dB} / 100 \mathrm{~m}$ to $-40 \mathrm{~dB} / 100 \mathrm{~m}$, and new fibers presenting considerably stronger crosstalk have been reported $[14](-7 \mathrm{~dB} / 100 \mathrm{~m})$. In the strong coupling regime, the nonlinear interactions between the modes can be averaged with respect to the random linear evolution of the field within each mode, since the linear evolution is significantly faster than the nonlinear evolution, as shown in [5],[6]. In this case, for $M$ modes with 2 polarizations, the nonlinear terms average to $-\mathrm{j} \Gamma|\vec{E}|^{2} \vec{E}$, where $\Gamma=4 / 3 \cdot(2 M) /(2 M+1) \cdot \bar{\gamma}$ with $\bar{\gamma}$ equal to the average nonlinear coefficient [6]. Moreover, the $\Gamma$ coefficient can be introduced to (2) in place of $\gamma_{a b c d}$.

The nonlinear noise power was calculated for a range of different linear coupling strengths ranging from the strong to the weak coupling regimes using a numerical model which divides the fiber in multiple sections of length, each with a constant random displacement of the core center position [7]. The coupling strength (XT) was quantified as the ratio between the power lost to the other modes from the $\mathrm{LP}_{01}$ mode and the power that remains in the $\mathrm{LP}_{01}$ mode. The nonlinear signal field $E_{d s}$ generated after a length $L$ is found by adding (1) over all fiber segments, while the interfering waves $a p, b q, c r$ are linearly propagated (attenuation, dispersion and linear mode coupling). As for dispersion managed single mode fiber systems, power depletion due to the FWM and higher-order mixing terms are neglected [15]. Finally, the total nonlinear noise power generated at the center of the WDM versus the WDM bandwidth is obtained by summing over the frequency offsets that satisfy $\omega_{p}+\omega_{q}=\omega_{r}+\omega_{s}$.

Fig. 2 shows the nonlinear noise power generated at the center of the WDM band versus the WDM bandwidth for the fiber presented in Table 1 and for different $X T$ values ranging from $-70 \mathrm{~dB} / 100 \mathrm{~m}$ to $-20 \mathrm{~dB} / 100 \mathrm{~m}$. The $X T$ value was set by varying the amplitude of the random variation in lateral section offset assuming a section length of $10 \mathrm{~m}$ (smaller section lengths generated similar results). The simulation results in Fig. 2 lay between two analytical lines obtained with (2) using: the ordinary fiber nonlinear coefficients (for zero, or weak mode coupling) [5], dotted line, and using the average nonlinear coefficients derived in [6] for strong mode coupling, dashed line. 


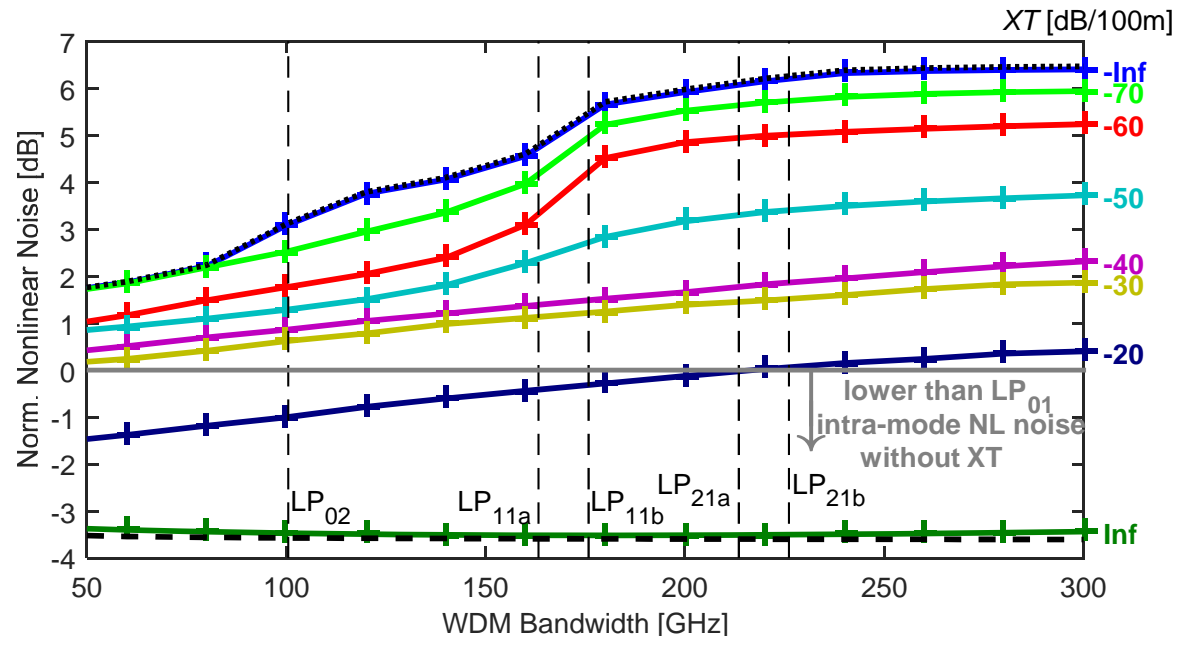

Fig. 2. Total nonlinear noise power in the $\mathrm{LP}_{01}$ mode as a function of WDM bandwidth showing analytical predictions from strong (dashed) and weak (dotted) regimes along with numerical simulations (solid) for different mode coupling strengths (colors).

It can be noted in Fig. 2 that the rate of decrease of the nonlinear noise as the coupling strength is increased is higher for higher bandwidths than for smaller bandwidths, which shows that the averaging of the nonlinear coefficients among the higher-order modes occurs more rapidly. For small values of $X T,-70$ and $-60 \mathrm{~dB} / 100 \mathrm{~m}$ the steps associated with the inter-mode interactions of $\mathrm{LP}_{01}$ with $\mathrm{LP}_{02}$ and $\mathrm{LP}_{21 \mathrm{a}} / \mathrm{LP}_{21 \mathrm{~b}}$, become smooth, but the step associated with $\mathrm{LP}_{11 \mathrm{a}} / \mathrm{LP}_{11 \mathrm{~b}}$ remains unchanged. This is in line with the asymmetries on the coupling strength between pairs of modes from the same mode groups (stronger) and from different mode groups (weaker) (modes $\mathrm{LP}_{02}$ and $\mathrm{LP}_{21 \mathrm{a}} / \mathrm{LP}_{21 \mathrm{~b}}$ belong to the same mode group). Increasing $X T$ up to $-40 \mathrm{~dB} / 100 \mathrm{~m}$, smooths the step associated with inter-mode interactions of $\mathrm{LP}_{01}$ with $\mathrm{LP}_{02}$. Furthermore, increasing $X T$ above $-20 \mathrm{~dB} / 100 \mathrm{~m}$ reduces nonlinear noise power below the $\mathrm{LP}_{01}$ intra-mode nonlinear noise power in the absence of linear coupling which was used to normalize the results. In the limit, strongly coupling all modes, using unitary matrices every $10 \mathrm{~m}$ (and shorter steps, as verified), the nonlinear noise power matches the analytical results (dashed line) obtained using (2) and the average nonlinear coefficients in [6].

\section{Conclusions}

For the crosstalk values shown by the majority of FMFs (from -50 to $-20 \mathrm{~dB} / 100 \mathrm{~m}$ ), the nonlinear noise is not accurately estimated by either the weak linear coupling regime or the strong coupling regime. However, the overall conclusion that the stronger coupling reduces nonlinear noise power remains valid. We have assessed for the first time the level of intermediate coupling required to minimize nonlinear noise in coupled few-mode propagation below that of uncoupled single-mode propagation. We observe a reduction of nonlinear noise below that of uncoupled singlemode propagation for linear coupling values above $-20 \mathrm{~dB} / 100 \mathrm{~m}$.

This work has been partially supported by the European Communities 7th Framework Program (Grants 619732INSPACE and 627545-SOLAS) and by the EPSRC (Grant EP/L000091/1-PEACE).

\section{References}

[1] D. Marcuse, Theory of Dielectric Optical Waveguides, 2nd ed. New York: Academic, 1991.

[2] K. Ho, et al., "Statistics of group delays in multimode fiber with strong mode coupling," J. Lightw. Technol., 29(21), p 3119, (2011).

[3] R.-J. Essiambre, et al., "Inter-modal nonlinear interactions between well separated channels in spatially-multiplexed fiber transmission," Proc. ECOC, paper Tu.1.C.4, (2012).

[4] F. Poletti, et al., "Description of ultrashort pulse propagation in multimode optical fibers," J. Opt. Soc. Am. B 25, p.1645, (2008).

[5] S. Mumtaz, et al., "Nonlinear Propagation in Multimode and Multicore Fibers: Generalization of the Manakov Equations," J. Lightw. Technol., 31(3), p.398, (2013).

[6] A. Mecozzi, et al., "Nonlinear propagation in multi-mode fibers in the strong coupling regime," Opt. Express 20, pp.11673, (2012).

[7] F. Ferreira, et al., "Nonlinear Semi-Analytical Model for Simulation of Few-Mode Fiber Transmission," Photon. Technol. Lett., 24(4), p.240, (2012).

[8] Y. Xiao, et al., "Theory of intermodal four-wave mixing with random linear mode coupling in few-mode fibers," Opt. Express 22, p. 32039 (2014).

[9] A. Ellis, et al., "Expressions for the nonlinear transmission performance of multi-mode optical fiber," Opt. Express 21, p.22834, (2013)

[10] G. Rademacher, et al., "Analytical description of cross modal nonlinear interaction in mode multiplexed multi-mode fibers," Photon. Technol. Lett. 24(21), pp.1929, (2012).

[11] F. Ferreira, et al., "Design of few-mode fibers with M-modes and low differential mode delay," J. Lightw. Technol.,32(3),p.353, (2014)

[12] O. Sinkin, et al., "Impact of broadband four-wave mixing on system characterisation," Proc. OFC, paper OTh3G, (2013).

[13] L. Grüner-Nielsen, et al., "Few mode transmission fiber with low DGD, low mode coupling, and low loss," J. Lightw. Technol., 30(23), p.3693, (2012).

[14] N. Fontaine, et al., "Experimental investigation of crosstalk accumulation in a ring-core fiber," Proc. PSSTMS, TuC4.2, Waikoloa, (2013).

[15] K. Inoue, "Phase-mismatching characteristic of four-wave mixing in fiber lines with multistage optical amplifiers," Opt. Lett. 17, p.801, (1992). 